> Le lysosome fut découvert en 1955 par Christian de Duve. La première démonstration par Hers d'un lien entre un déficit enzymatique et une maladie de surcharge (maladie de Pompe) ouvrit la voie à une série de découvertes essentielles qui ont culminé avec le traitement de la maladie de Gaucher par perfusions de $\beta$-glucosidase au début des années 90 . Aujourd'hui la thérapie enzymatique recombinante est une réalité pour la maladie de Fabry, la mucopolysaccharidose de type I (MPS I) et la mucopolysaccharidose de type VI (MPS VI). Chez les patients atteints de MPS I, la laronidase ( $\alpha$-L-iduronidase humaine recombinante) améliore significativement la fonction respiratoire et les capacités physiques, réduit l'hépatomégalie et l'accumulation de glycosaminoglycanes et a un profil de sécurité favorable. À la suite des résultats positifs des essais de phase I et de phase II, un essai clinique de phase III contrôlé en double-aveugle contre placebo fut conduit pour la galsulfase (arylsulfatase B humaine recombinante) chez des patients atteints de MPS VI. Les résultats de cette étude confirmèrent la sécurité et l'efficacité de la galsulfase qui améliora significativement le test de marche de 12 minutes et réduisit l'excrétion urinaire de dermatane sulfate. L' $\alpha$-glucosidase humaine recombinante a fait l'objet d'essais cliniques pour la thérapie de la maladie de Pompe. Les données cliniques montre que l'enzyme nettoie efficacement le glycogène du muscle cardiaque et des fibres de type I du muscle squelettique, mais pas les fibres de type II. Des essais cliniques avec des enzymes recombinantes sont en cours pour la MPS II, et sur le point de débuter pour la maladie de Niemann-Pick de type B. Plusieurs défis persistent cependant pour les thérapies enzymatiques substitutives, en particulier le traitement du système nerveux central. Des stratégies alternatives, telles que la réduction de substrat ou l'emploi de petites molécules chaperons pour restaurer des enzymes mutantes, mal repliées ou instables, mais qui ont gardé une fonction résiduelle, sont en cours d'évaluation. <

\section{Thérapies enzymatiques substitutives des maladies lysosomales}

\section{Dominique P. Germain}

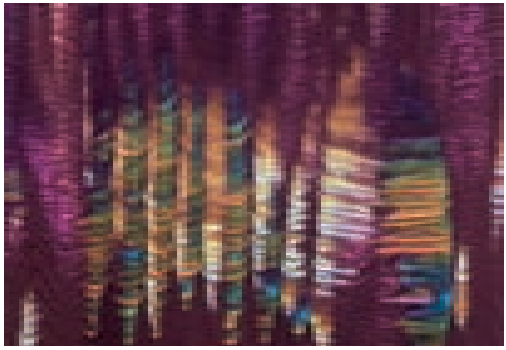

Unité de Génétique Clinique, Hôpital Européen Georges Pompidou, 20, rue Leblanc, 75015 Paris, France.

dominique.germain@egp.aphp.fr

Les maladies lysosomales sont un groupe de plus de 50 maladies héréditaires du métabolisme. Dès lors que l'on démontra que ces maladies lysosomales étaient dues à des déficits enzymatiques [1-3], il devenait logique d'envisager une thérapie substitutive en apportant l'enzyme exogène pour traiter ces pathologies.

La première maladie pour laquelle furent réalisés les travaux pionniers de thérapie enzymatique substitutive fut la maladie de Gaucher. Les leçons de la maladie de Gaucher servent aujourd'hui au développement d'enzymothérapies substitutives pour d'autres maladies lysosomales: la maladie de Fabry qui fait l'objet de ce numéro thématique, mais aussi plus récemment la mucopolysaccharidose de type 1 (maladie de Hurler-Scheie et maladie de Scheie), la mucopolysaccharidose de type 6 (maladie de Maroteaux-Lamy) et la glycogénose de type 2 (maladie de Pompe), tandis que des espoirs légitimes d'apparition à court terme de traitements spécifiques existent pour la mucopolysaccharidose de type 2 (syndrome de Hunter) et la maladie de Niemann-Pick de type B qui seront brièvement décrites dans cette revue générale.

\section{Maladie de Gaucher}

Il s'agit d'une affection décrite dans sa thèse de médecine il y a plus d'un siècle, par un médecin français, Philippe Gaucher, qui pensait initialement avoir décrit un épithélioma de la rate chez l'une de ses patientes. La maladie de Gaucher est, comme la maladie de Fabry, une sphingolipidose. Elle est due à l'atteinte du gène GBA, situé sur le 
chromosome 1 , et a une transmission génétique autosomique récessive. II existe un déficit en $\beta$-glucosidase acide, qui va conduire à l'accumulation de glucocérébroside, encore appelé glucosylcéramide (Gbl). Cette maladie est panethnique mais a la particularité d'être beaucoup plus fréquente dans certaines ethnies et est par exemple la maladie génétique la plus fréquente dans la communauté juive ashkénaze. La fréquence est d'environ un cas pour 100000 personnes dans la population générale tandis qu'elle est d'un cas pour 1000 chez les Ashkénazes [4].

Les cellules cibles de la maladie sont les phagocytes mononucléés, monocytes et macrophages, constituant l'ancien «système réticuloendothélial». L'atteinte de ces cellules va expliquer la distribution des symptômes dans les organes ou les monocytes et les macrophages sont nombreux. Une splénomégalie est ainsi présente dans $90 \%$ des cas. Sont également au premier plan, l'hépatomégalie, l'envahissement de la moelle osseuse par des cellules de surcharge, correspondant aux précurseurs des macrophages, qui déplacent les autres lignées. Ce déficit sur les trois lignées de l'hématopoïèse sera responsable au niveau périphérique d'une thrombopénie, une anémie et une leucopénie, susceptibles d'être aggravées par un hypersplénisme. Les malades sont asthéniques. L'envahissement de la moelle osseuse retentit également sur la structure et la morphologie de l'os avec apparition de douleurs osseuses, de trouble du remodelage, de nécroses avasculaires et de fractures. Dans l'immense majorité des cas, il n'existe pas d'atteinte du système nerveux central, mais dans les cas où le déficit enzymatique est très profond, les macrophages ne parviennent plus à dégrader le Gbl issu de leur propre métabolisme endogène, et une atteinte du système nerveux central s'associe alors aux manifestations viscérales [5].

Comparativement aux essais thérapeutiques aujourd'hui exigés par les agences de régulation préalablement à l'autorisation de mise sur le marché d'un nouveau médicament, la rapidité avec laquelle le processus a été conduit pour la maladie de Gaucher est assez singulière. Un essai préliminaire avec une enzyme extractive non modifiée fut interrompu en l'absence de résultats thérapeutiques probants. Les avancées ont consisté à tirer parti des connaissances acquises sur un récepteur présent à la surface des macrophages: le récepteur mannose [6]. Le fait de modifier l'enzyme native, extraite de placenta, et d'exposer les terminaisons mannose des chaînes oligosaccharidiques de la $\beta$-glucosidase acide, a permis de favoriser la liaison de la glycoprotéine au récepteur mannose présent à la surface des macrophages, à l'origine d'une endocytose ligand-dépendante et de l'aider à être internalisée dans les macrophages et à gagner le lysosome où le pH acide est nécessaire à l'activité catalytique de l'enzyme et à son efficacité thérapeutique [7]. L'enzyme extractive modifiée (alglucérase) fut approuvée après un essai thérapeutique en ouvert, ayant porté uniquement sur 12 malades. La présence d'un enfant de plus petit poids corporel parmi les malades a permis de constater l'existence d'un effet dose. L'alglucérase a été approuvée il y a une quinzaine d'années. En raison de risques, jamais vérifiés, de transmission d'agents pathogènes tels que les prions, une substitution de l'alglucérase, d'origine placentaire, par l'imiglucérase (Cerezyme ${ }^{\circledR}$ ), enzyme recombinante produite par génie génétique dans des cellules d'ovaires de hamster chinois (cellules $\mathrm{CHO}$ ), eut lieu en 1996. L'efficacité thérapeutique de l'enzyme recombinante est comparable à celle de l'enzyme d'origine extractive [8]. Plus de 4500 patients sont aujourd'hui traités avec succès par imiglucérase à travers le monde. Dans la maladie de Gaucher, l'efficacité thérapeutique de l'enzymothérapie sur l'asthénie, l’hépatosplénomégalie, la thrombopénie et l'anémie est remarquable. Un certain nombre d'interrogations subsistent sur la réponse de l'os à l'enzymothérapie, qui nécessite souvent plusieurs années de traitement et reste parfois incomplète $[9,10]$. Certaines questions sont intéressantes à discuter, comme la dose de maintenance adéquate et la fréquence optimale d'administration de l'imiglucérase [11]. II ne faut pas oublier que, même si ce produit est très efficace, il a un coût pour la société à travers l'assurance maladie. Il est donc légitime de rechercher le meilleur rapport entre son efficacité et son prix [12].

D'autres voies thérapeutiques émergent actuellement pour le traitement de la maladie de Gaucher. II sera intéressant de suivre les résultats à long terme de la réduction de substrat [13] ainsi que des molécules chaperons, approches différentes de l'enzymothérapie qui reste le traitement de première intention de la maladie de Gaucher pour laquelle elle a représenté une avancée scientifique majeure qui demeure un modèle aujourd'hui appliqué à d'autres maladies lysosomales.

\section{Mucopolysaccharidose de type I (MPS I)}

Cette maladie a été décrite pour la première fois dans sa forme sévère par Gertrud Hurler en 1919. L'accumulation pathologique de mucopolysaccharides ou glycosaminoglycanes fut démontrée beaucoup plus tard. La MPS I est due à un déficit enzymatique en $\alpha$-L-iduronidase. Dans l'intervalle, avait été individualisée une affection, subséquemment attribuée au même déficit enzymatique [14], mais ayant une traduction clinique totalement différente, connue aujourd'hui sous le nom de maladie de Scheie.

La MPS I a un spectre clinique extrêmement étendu. Sa forme la plus grave (maladie de Hurler) est une affection dramatique, de révélation pédiatrique, dont il importe de faire le diagnostic précocement, car de la rapidité diagnostique vont dépendre les possibilités thérapeutiques représentées par la greffe de moelle osseuse [15] et l'enzymothérapie [16]. L'examen clinique à la naissance est le plus souvent normal à l'exception d'une possible hernie inguinale ou ombilicale, signe de valeur si le nourrisson n'est pas un prématuré. Vont ensuite progressivement apparaître un certain nombre de signes, souvent non spécifiques, tels qu'un encombrement rhinopharyngé avec des infections de la sphère $O R L$ ou respiratoire. Une cyphose et une scoliose dorsolombaire peuvent se développer lors de l'acquisition de la station assise. Les enfants sont, de ce fait, 
volontiers vus initialement soit dans un service d'ORL, soit dans un service de chirurgie orthopédique où il importe d'évoquer le diagnostic de MPS I. Avant l'âge de deux ans, apparaissent des signes plus spécifiques, au premier rang desquels la dysmorphie faciale, une hypotonie de l'abdomen et des hernies qui ont la particularité de volontiers récidiver après cure chirurgicale. L'avance staturo-pondérale, initialement observée, va s'inverser avec cassure de la courbe de croissance aboutissant à un nanisme. L'atteinte respiratoire, par surcharge des muqueuses en glycosaminoglycanes, va conduire à des apnées du sommeil, puis à l'insuffisance respiratoire. L'atteinte cardiaque peut comporter une composante valvulaire, mais aussi une hypertension artérielle pulmonaire, une hypertension artérielle systémique et une myocardiopathie. Un enraidissement articulaire va progressivement s'installer avec attitude en flexum des coudes et des genoux, aspect de main en griffe et réduction considérable de la mobilité des épaules. Les dépôts de glycosaminoglycanes dans les organes sensoriels sont responsables d'opacités cornéennes et d'une atteinte auditive (surdité mixte). Au niveau cérébral, les dépôts d'héparane-sulfate vont conduire à une régression des acquisitions de l'enfant et à un retard psychomoteur.

Il existe des cas, moins nombreux, de maladie de Scheie (Figure 1). Il est sans doute artificiel, comme pour les variants cardiaques et rénaux de la maladie de Fabry, de vouloir séparer en deux affections distinctes les deux pôles d'un large spectre clinique avec, au milieu, le «phénotype Hurler-Scheie», qui emprunte des manifestations cliniques à la forme la plus grave et à la forme dite atténuée.

Le premier essai clinique pour la mucopolysaccharidose de type 1 fut conduit par Emil Kakkis, dans le groupe d'Elisabeth Neufeld [17]. Cet essai monocentrique a porté sur 10 malades, pour la plupart atteints de la forme intermédiaire ou maladie de Hurler-Scheie (MPSIHS) car, les enzymes recombinantes ne pouvant pas franchir la barrière hématoencéphalique, des résultats thérapeutiques modestes sont attendus en cas d'atteinte du système nerveux central avec retard mental, comme c'est le cas dans la maladie de Hurler (MPS IH). Les perfusions de laronidase furent hebdomadaires et non bi-mensuelles. Cet essai visait à montrer la bonne tolérance et l'innocuité de la nouvelle molécule, mais aussi à s'intéresser aux premiers résultats thérapeutiques évalués par la réduction de la taille du foie et de la rate et la baisse de l'excrétion urinaire excessive de glycosaminoglycanes (héparane sulfate et dermatane sulfate). Les résultats furent favorables avec diminution sous traitement de l'excrétion urinaire des glyco- saminoglycanes, accompagnée d'une réduction de la splénomégalie ainsi que du volume hépatique mesurés par échographie. L'amplitude d'abduction des épaules s'est par ailleurs accrue participant à une amélioration de la qualité de vie de l'enfant [17].

Un essai international multicentrique (cinq centres répartis dans trois pays) a ensuite inclus 45 malades âgés de plus de cinq ans atteints de MPS I. La moitié des patients reçut la laronidase et l'autre moitié un placebo en perfusion hebdomadaire. Les critères primaires d'efficacité étaient la capacité vitale forcée, qui fut statistiquement améliorée, et le test de marche pendant six minutes lors duquel le périmètre de marche fut testé avant et après traitement. Pendant la période en doubleaveugle de l'essai, le groupe traité gagna 19 mètres, alors que le groupe placebo continua de se dégrader. Après un an de traitement, la progression du périmètre de marche fut de 41 mètres pour le groupe traité. Des critères secondaires ou tertiaires furent analysés. La diminution de l'excrétion urinaire des glycosaminoglycanes fut confirmée (moins $54 \%$ à six mois et moins $65 \%$ à un an) de même qu'une réduction modérée du volume hépatique, une diminution du nombre d'apnées du sommeil (moins six événements par heure à six mois) et une petite amélioration de la raideur articulaire des épaules [16].

La laronidase (Aldurazyme ${ }^{\circledast}$ ), a obtenu l'autorisation de mise sur le marché aux États-Unis et en Europe en 2003. II n'y a pas eu d'exigence d'études complémentaires de la part des agences de régulation (Food and Drug Administration, FDA et European Agency for the Evaluation of Medicinal Products, EMEA), qui ont considéré que les données de l'essai international de phase 3 en double aveugle étaient de qualité [18]. En revanche, il a été demandé au laboratoire Genzyme de continuer un programme de surveillance clinique rigoureux et de suivre l'efficacité thérapeutique au long cours de l'enzymothérapie. Les agences ont par ailleurs enjoint au laboratoire de tester l'efficacité

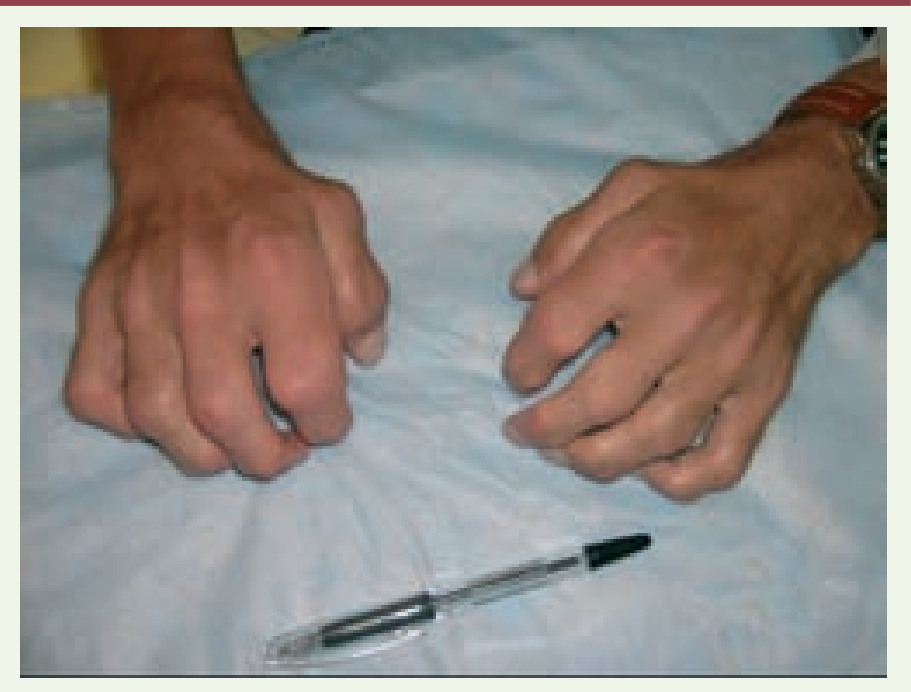

Figure 1. Déformations articulaires (« mains en griffe ») chez un patient atteint d'une mucopolysaccharidose de type I (maladie de Scheie) (iconographie : Dr Nathalie Guffon, Lyon, France). 
de l'enzyme sur les enfants âgés de moins de cinq ans, précédemment exclus des essais cliniques.

Chez les enfants très jeunes, atteints de maladie de Hurler, l'enzyme ne passe pas la barrière hémato-méningée et il existe une alternative thérapeutique, la greffe de moelle osseuse qui, à condition qu'elle soit réalisée très tôt, donne de bons résultats thérapeutiques [15] mais est accompagnée d'une morbi-mortalité lourde et pose des problèmes de recrutement des donneurs. À ce jour, la thérapie enzymatique substitutive est généralement privilégiée au-dessus de deux ans, tandis que la greffe reste en première intention pour les malades âgés de moins de deux ans, atteints de la forme très sévère de l'affection.

\section{Mucopolysaccharidose de type VI (MPS VI)}

La mucopolysaccharidose de type VI (MPS VI) ou maladie de Maroteaux-Lamy a été décrite par deux pédiatres français, Pierre Maroteaux et Maurice Lamy, en 1963. L'enzyme déficitaire est l'arylsulfatase $\mathrm{B}$ ou $\mathrm{N}$-acétylgalactosamine-4-sulfatase [19]. Il y a accumulation dans l'organisme de glycosaminoglycanes (dermatane sulfate) mais, à la différence de la MPS I, l'absence de dépôts d'héparane-sulfate, explique vraisemblablement l'absence de régression psychomotrice chez ces enfants.

La maladie de Maroteaux-Lamy (Figure 2) dans sa forme grave présente un tableau clinique multisystémique avec une morbi-mortalité précoce, mais sans régression des acquisitions. À l'opposé du spectre clinique, il existe une forme relativement atténuée, avec des symptômes permettant une survie à l'âge adulte.

Le diagnostic est confirmé par la mise en évidence du déficit enzymatique en arylsulfatase $B$ dans les leucocytes ou la mise en évidence de l'excrétion urinaire de dermatane sulfate. II existe des inclusions des polynucléaires sur le frottis sanguin.

Comme dans la maladie de Hurler-Scheie, la greffe de moelle osseuse peut donner des résultats intéressants dans la maladie de MaroteauxLamy [20]. Les indications sont l'existence d'une cardiomyopathie ou d'apnées du sommeil sévères. La greffe prolonge la survie avec amélioration de la qualité de vie, de l'atteinte cardiaque, de la viscéromégalie et de la dysmorphie faciale. En revanche, ses résultats sur l'atteinte osseuse sont mauvais.

Dans un premier essai thérapeutique contrôlé, randomisé, en doubleaveugle, portant sur six malades, les auteurs ont testé l'enzyme recombinante en perfusion hebdomadaire en étudiant deux doses: $1 \mathrm{mg} / \mathrm{kg}$ de poids et $0,2 \mathrm{mg} / \mathrm{kg}$ de poids. Les conclusions de cet essai de phase 1 montrèrent une bonne tolérance de l'arylsulfatase $B$ recombinante, une réduction de l'excrétion urinaire de dermatane sulfate et une amélioration du test de marche pendant six minutes, plus notable pour les patients les plus sévèrement touchés et à la posologie de 1 mg/kg [21]. Un essai de phase 2 portant sur 10 malades fut ensuite organisé. Seule la posologie de $1 \mathrm{mg} / \mathrm{kg}$ fut conservée dans cette étude, qui confirma la bonne tolérance de l'enzyme avec toutefois survenue d'un certain nombre d'effets indésirables graves, non liés au traitement dans l'opinion des investigateurs. Une séroconversion fut observée comme lors des traitements de la maladie de Fabry et de la maladie de Gaucher, apparemment sans baisse d'efficacité thérapeutique. L'analyse des critères d'efficacité montra une réduction de l'excrétion urinaire des glycosaminoglycanes, une amélioration de $139 \%$ du test de marche de 12 minutes et une amélioration du test de montée des escaliers pendant 3 minutes. L'absence de groupe contrôle et de placebo diminuait toutefois la valeur de ces résultats [22].

Un troisième essai, multicentrique, randomisé, fut donc mis en place, avec une première période en doubleaveugle, contre placebo d'une durée de 6 mois pendant laquelle 19 malades furent traités par l'enzyme recombinante et 20 malades traités par placebo. Les résultats obtenus furent remarquables avec amélioration statistiquement significative du test de marche (92 mètres de différence moyenne entre les groupes traités et non traités) dans cette étude. La réduction de l'excrétion urinaire du dermatane sulfate fut également statistiquement significative, à la différence du test de montée des escaliers pendant trois minutes qui montrait une tendance favorable, avec une différence moyenne de six marches par minute entre les groupes traité et non traité, sans toutefois atteindre la significativité statistique.

L'autorisation de mise sur le marché aux États-Unis a été accordée le $1^{\text {er }}$ juin 2005 pour Naglazyme ${ }^{\circledast}$ dont la

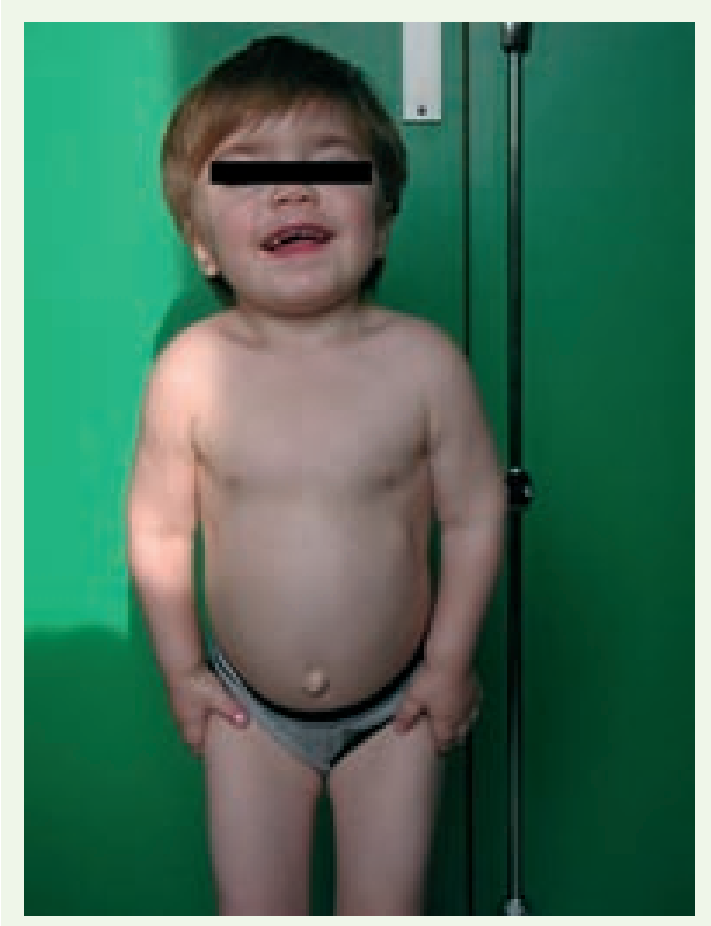

Figure 2. Mucopolysaccharidose de type VI (maladie de Maroteaux-Lamy) chez une enfant âgée de 5 ans (iconographie: Dr Nathalie Guffon, Lyon, France). 
dénomination commune internationale est galsulfase, désormais disponible sur le marché américain. La Food and Drug Administration (FDA) américaine a demandé au laboratoire BioMarin d'étudier l'effet de Naglazyme ${ }^{\circledast}$ sur la dysplasie osseuse des patients de moins d'un an et d'assurer un suivi rigoureux du devenir des patients sous traitement. $\varepsilon$ n revanche, aucune demande d'étude complémentaire n'a été formulée, les essais disponibles ayant été bien conduits [23].

L'autorisation de mise sur le marché n'est pas encore disponible dans l'Union européenne mais le COMP (Committee for Orphan Medicinal Products) de l'agence européenne (European Medicines Agency, EMA) a délivré une opinion positive en septembre 2005 avec la décision finale de la commission européenne attendue en 2006. En France, les premières autorisations temporaires d'utilisation ont été accordées par I'Agence Française de Sécurité Sanitaire des Produits de Santé (AFSSAPS).

\section{Maladies de Niemann-Pick de type A et B}

II existe dans les maladies de Nieman-Pick de types A et B un déficit en sphingomyélinase acide lysosomale. Lorsque le déficit enzymatique est profond, sous-tendu par des mutations particulièrement délétères, un retard psychomoteur est présent, définissant la maladie de Nieman-Pick de type A. La maladie de Niemann-Pick de type B, probablement sous-diagnostiquée, débute dans l'enfance par une hépatosplénomégalie qui a tendance à devenir moins visible lorsque le malade grandit et que le volume corporel augmente, masquant plus ou moins cette viscéromégalie. À l'âge adulte, vont apparaître des perturbations du profil lipidique et surtout une atteinte pulmonaire, cliniquement manifeste, visualisée sous la forme d'un infiltrat réticulonodulaire sur la radio et le scanner thoraciques.

L'enzyme recombinante a été fabriquée in vitro au laboratoire et des essais précliniques ont été conduits sur le modèle murin (souris $a s m^{-/}$). II semble exister des difficultés pour l'internalisation de l'enzyme recombinante dans les macrophages pulmonaires et il y a lieu d'améliorer l'adressage de l'enzyme vers sa cible: le lysosome [24]. L'histoire naturelle de cette maladie est parallèlement étudiée dans un registre qui devrait permettre de mieux apprécier, l'efficacité d'une future thérapie enzymatique substitutive pour le type B par comparaison avec cette population témoin.

\section{Glycogénose de type II ou maladie de Pompe}

La première maladie lysosomale décrite n'était ni une mucopolysaccharidose ni une sphingolipidose, mais une glycogénose: la maladie de Pompe ou glycogénose de type 2 due à un déficit en maltase acide ou $\alpha$-glucosidase lysosomale [1]. Deux grands phénotypes sont observés: la forme infantile, ou maladie de Pompe proprement dite, constamment mortelle avant l'âge de trois ans, et des formes adultes dans lesquelles les patients ont une activité enzymatique résiduelle modifiant considérablement le phénotype clinique. Entre ces deux pôles du spectre clinique existent des formes intermédiaires, dites juvéniles, apparemment sous-diagnostiquées. La maladie de Pompe est une affection de révélation pédiatrique, constamment mortelle avant l'âge de 3 ans en l'absence de traitement spécifique [25]. Elle est caractérisée par une hypotonie extrême et une cardiomégalie considérable avec traduction électrocardiographique, radiographique (Figure 3) et clinique. À l’âge adulte, la glycogénose de type II se présente comme une myopathie avec, pour certains patients, des signes relativement discrets tels qu'un décollement scapulaire et un défaut des masses musculaires paravertébrales. Les formes de l'adulte peuvent s'accompagner d'une insuffisance respiratoire sévère nécessitant la trachéotomie et la ventilation assistée.

Dans le cas de la maladie de Pompe infantile, plusieurs essais thérapeutiques ont été conduits avec des enzymes provenant de trois systèmes de production différents. Un premier essai clinique original fut conduit par l'équipe d'Arnold Reuser, qui avait développé la production d' $\alpha$-galactosidase dans le lait de lapine transgénique en association avec le laboratoire Pharming [26]. La production de très larges quantités d'enzymes semblait toutefois difficile dans ce système et des enzymes recombinantes ont ensuite été produites en cellules d'ovaires de hamster chinois (cellules $\mathrm{CHO}$ ) d'abord par le laboratoire Synpac, puis par le laboratoire Genzyme [27].

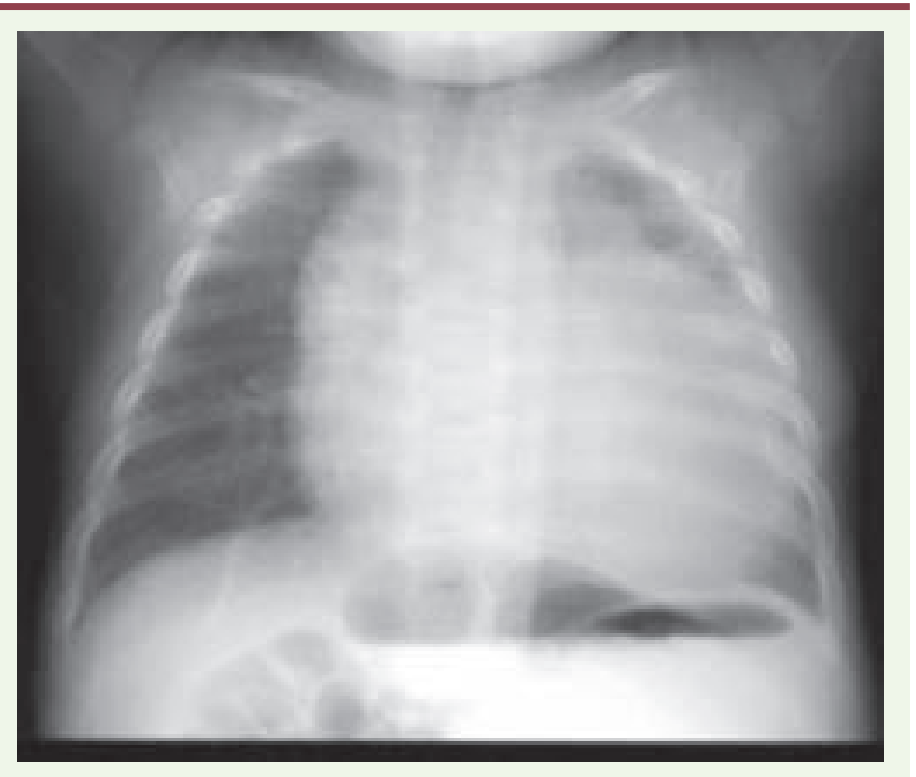

Figure 3. Cardiomégalie chez un nourrisson atteint de déficit en $\alpha$-glucosidase (maladie de Pompe) (iconographie: Dr François Sassolas et Pr Marc Nicolino, Lyon, France). 
Un essai pilote conduit à l'université de Duke, en Caroline du Nord, aux États-Unis et utilisant l'enzyme recombinante débuta en 1999. Trois patients furent inclus dans cet essai dont l'objectif primaire était la survie sans ventilation assistée, puisque cette maladie est constamment mortelle avant l'âge de 3 ans en l'absence de traitement. La réduction de la taille du ventricule gauche et une modification du score de développement de ces jeunes patients étaient les critères secondaires. Les effets sur la cardiomyopathie furent excellents, avec réduction nette de la masse cardiaque. Les résultats furent beaucoup plus hétérogènes sur le score de développement moteur pour lequel seul un patient parvint à rattraper les valeurs normales. L'université de Duke compléta son étude en incluant huit malades supplémentaires pour lesquels différentes doses furent étudiées. Les résultats furent très hétérogènes avec trois patients survivants (alors qu'ils n'auraient pas dû l'être compte tenu de l'extrême sévérité de la maladie de Pompe). Cependant, huit décès furent à déplorer. Les facteurs responsables de cette hétérogénéité sont mal compris même si l'on considère qu'un début trop tardif du traitement est un facteur de mauvais pronostic.

Deux essais furent également conduits à l'Université de Rotterdam, le premier portant sur quatre malades atteints de forme infantile. Les résultats furent bons puisque les malades survécurent au-delà de quatre ans. Cependant, les deux patients ayant commencé le traitement un peu plus tardivement décédèrent ensuite, tandis que les deux autres patients sont désormais sous ventilation assistée. Un autre essai, portant sur trois patients atteints de forme dite juvénile, donna des résultats très favorables [28].

Il existe de nombreux défis non résolus à ce jour pour la maladie de Pompe. Quel(s) facteur(s) différencie(nt) les bons des mauvais répondeurs au niveau du muscle squelettique [29] ? Quel est le rôle du statut immunologique (CRIM positif / CRIM négatif.) ? Quelle est la dose d' $\alpha$-glucosidase recombinante optimale à administrer? La question de la production en quantité suffisante de l'enzyme pourrait se poser, puisque les doses perfusées sont considérables (10, 20, voire $40 \mathrm{mg}$ de protéines par kilo de poids). Dans le même temps, de nombreux patients adultes sont par ailleurs actuellement en déshérence de soins avec l'espoir de recevoir un jour cette thérapeutique spécifique disponible pour les enfants. Cependant, si l'on exclut l'essai portant sur les trois patients atteint de forme juvénile, il n'existe à ce jour aucune donnée contrôlée démontrant un bénéfice thérapeutique de l'enzyme pour les formes juvéniles ou adultes de la maladie.

Pour essayer de répondre à ces questions, deux essais complémentaires ont été initiés. Le premier (AGLU-01602, promoteur: Genzyme) est un essai international, s'adressant aux patients atteints de la forme infantile de maladie de Pompe, âgés de moins de six mois et porteurs d'une cardiomyopathie. Le critère primaire était la survie à 18 mois sans ventilation assistée. Une deuxième étude, (AGLU-01702, promoteur: Genzyme), a été menée pour inclure les patients qui ne satisfaisaient pas aux critères de l'essai AGLU-01602 ; elle s'adresse à des patients légèrement plus âgés. Les résultats sont en cours d'analyse. Enfin, sur les formes adultes de glycogénose de type II, un registre a suivi l'histoire naturelle de la maladie pendant un an, dans une pre- mière phase observationnelle, avant d'étudier l'efficacité clinique de la maltase acide recombinante.

\section{Conclusions}

Le développement et la mise sur le marché des thérapies enzymatiques a évolué depuis les travaux pionniers réalisés pour la maladie de Gaucher. Un essai clinique en ouvert sur 12 malades avait alors conduit à l'autorisation de mise sur le marché. Les exigences des agences de régulation sont aujourd'hui nettement plus élevées, obligeant les promoteurs et les investigateurs à mettre au point des programmes de développement et des essais cliniques de plus en plus complexes pour démontrer l'efficacité clinique des nouvelles thérapies, rapprochant le développement de ces médicaments orphelins des programmes établis pour les médicaments classiques. La disponibilité d'une thérapie enzymatique substitutive pour cinq affections est porteuse d'espoirs pour les autres maladies lysosomales, d'autant que des traitements pour la mucopolysaccharidose de type 2 (syndrome de Hunter) et la maladie de Nieman-Pick B devraient prochainement apparaître. Le prochain défi pour le traitement des maladies de surcharge des lysosomes est le système nerveux central, inaccessible aux enzymes du fait de la barrière hémato-encéphalique. Ce défi sera peut-être levé par la thérapie génique ou d'autres approches telles que la réduction de substrat, ou les protéines chaperones dans un futur que l'on espère le plus proche possible pour les patients atteints de ces maladies orphelines. $\diamond$

\section{SUMMARY}

Enzyme replacement therapies for lysosomal storage disorders

The lysosome was discovered in 1955 by Christian de Duve. The first demonstration by Hers of a link between an enzyme deficiency and a storage disorder (Pompe disease) paved the way for seminal discoveries culminating in the successful treatment of Gaucher disease with beta-glucosidase. Today, enzyme replacement therapy is a reality for Fabry disease, mucopolysaccharidosis type I (MPS I) and mucopolysaccharidosis type VI (MPS VI). In patients with MPS I, laronidase (recombinant human $\alpha$-L-iduronidase) significantly improves respiratory function and physical capacities, reduces hepatomegaly and glycosaminoglycan storage, and has a favorable safety profile. Following positive results from phase I and phase II studies, a randomized, double-blind, multicentre phase III trial of galsulfase (recombinant human arylsulfatase B) was conducted in patients affected with MPS VI. Data from this study confirmed the safety and efficacy of galsulfase which significantly improved the 12-minutes walk test and reduced urinary dermatan sulftate. Recombinant 
human alpha-glucosidase (rhGAA) is currently in clinical trials for therapy of Pompe disease. Clinical data show that the enzyme efficiently clears glycogen from cardiac muscle and type I skeletal muscle fibers, but not type II fibers. Clinical trials with recombinant human enzymes are ongoing in MPS II and about to begin in Niemann-Pick B disease. Significant challenges remain for enzyme replacement therapies, particularly the treatment of central nervous system disease. Alternative strategies, such as substrate deprivation and enzyme enhancement therapy which employs small molecules as "pharmacological chaperones" to rescue misfolded and/ or unstable mutant enzymes that have residual function, are currently being investigated. $\diamond$

\section{RÉFÉRENCES}

1. Hers HG. Alpha-glucosidase deficiency in generalized glycogen storage disease (Pompe's disease). Biochem J 1963; 86 : 11-6.

2. Brady RO, Kanfer JN, Shapiro D. Metabolism of glucocerebrosides. II. Evidence of an enzymatic deficiency in Gaucher's disease. Biochem Biophys Res Commun $1965 ; 18: 221-5$.

3. Neufeld EF, Fratantoni JC. Inborn errors of mucopolysaccharide metabolism. Science $1970 ; 169$ : 141-6.

4. Mistry PK. Gaucher's disease : a model for modern management of a genetic disease. J Hepatol 1999; 30 (suppl 1) : 1-5.

5. Germain DP. Gaucher's disease : a paradigm for interventional genetics. Clin Genet 2004; 65: 77-86

6. Stahl P, Schlesinger PH, Sigardson $\varepsilon$, et al. Receptor-mediated pinocytosis of mannose glycoconjugates by macrophages : characterization and evidence for receptor recycling. Cell $1980 ; 19: 207-15$

7. Barton NW, Furbish FS, Murray GJ, et al. Therapeutic response to intravenous infusions of glucocerebrosidase in a patient with Gaucher disease. Proc Natl Acad Sci USA $1990 ; 87$ : 1913-6.

8. Grabowski G, Barton NW, Pastores G, et al. Enzyme therapy in type 1 Gaucher disease : comparative efficacy of mannose-terminated glucocerebrosidase from natural and recombinant sources. Ann Intern Med $1995 ; 122$ : 33-9.

9. Pastores $\mathrm{G}$, Weinreb NJ, Aerts $\mathrm{H}$, et al. Therapeutic goals in the treatment of Gaucher disease. Semin Hematol 2004 ; 41 : 4-14.

10. Weinreb NJ, Aggio MC, Andersson HC, et al. Gaucher disease type 1 : revised recommendations on evaluations and monitoring for adult patients. Semin Hematol $2004 ; 41: 15-22$.
11. Hollak CM, Aerts J, Goudsmit R, et al. Individualised low-dose alglucerase therapy for type 1 Gaucher's disease. Lancet $1995 ; 345$ : 1474-8.

12. Andersson $H C$, Charrow J, Kaplan $P$, et al. Individualization of long-term enzyme replacement therapy for Gaucher disease. Genet Med $2005 ; 7: 105-10$.

13. Cox TM, Lachmann R, Hollak C, et al. Novel oral treatment of Gaucher's disease with Nbutyldeoxynojirimycin (OGT 918) to decrease substrate biosynthesis. Lancet $2000 ; 355$ : 1481-5.

14. Bach G, Friedman R, Weissmann B, Neufeld EF. The defect in the Hurler and Scheie syndromes: deficiency of -L-iduronidase. Proc Natl Acad Sci USA 1972; 69 : 2048-51.

15. Guffon N, Souillet G, Maire I, et al. Follow-up of nine patients with Hurler syndrome after bone marrow transplantation. J Pediatr 1998; 133 : 119-25.

16. Wraith JE, Clarke LA, Beck M, et al. Enzyme replacement therapy for mucopolysaccharidosis I: a randomized, double-blinded, placebo-controlled, multinational study of recombinant human alpha-L-iduronidase (laronidase). J Pediatr 2004 ; 144 : 581-8.

17. Kakkis $\varepsilon$, Muenzer J, Tiller GE, et al. Enzyme-replacement therapy in mucopolysaccharidosis I. $N$ EnglJ Med $2001 ; 344: 182-8$.

18. Wraith JE, Hopwood J, Fuller M, et al. Laronidase treatment of mucopolysaccharidosis I. BioDrugs $2005 ; 19: 1-7$.

19. Litjens T, Brooks DA, Peters C, et al. Identification, expression, and biochemical characterization of $\mathrm{N}$-acetylgalactosamine-4-sulfatase mutations and relationship with clinical phenotype in MPS-VI patients. Am J Hum Genet 1996; 58 : 1127-34.

20. Herskhovitz $\varepsilon$, Young $\varepsilon$, Rainer J, et al Bone marrow transplantation for Maroteaux-Lamy syndrome (MPS VI) : long-term follow-up. J Inherit Metab Dis 1999; 22 : 50-62.

21. Harmatz P, Chester B, Whitley CB, et al. Enzyme replacement therapy in mucopolysaccharidosis VI (Maroteaux-Lamy syndrome). J Pediatr 2004 ; $144: 574-80$.

22. Harmatz $P$, Ketteridge D, Giugliani R, et al. Direct comparison of measures of endurance, mobility, and joint function during enzyme-replacement therapy of mucopolysaccharidosis VI (MaroteauxLamy syndrome) : results after 48 weeks in a phase 2 open-label clinical study of recombinant human $\mathrm{N}$-acetylgalactosamine 4-sulfatase. Pediatrics 2005 ; 115 : e681-9.

23. Galsulfase : arylsulfatase B, BM 102, recombinant human arylsulfatase $B$, recombinant human $N$ acetylgalactosamine-4-sulfatase, rhASB. Drugs RD $2005 ; 6: 312-5$.

24. Dhami GK, Babwah AV, Sterne-Marr R, Ferguson SS. Mannose 6-phosphate receptor-mediated uptake is defective in acid sphingomyelinase-deficient macrophages: implications for NiemannPick disease enzyme replacement therapy. J Biol Chem 2004 ; 279 : 1526-32.

25. Kishnani PS, Howell RR. Pompe disease in infants and children. J Pediatr 2004 ; 144 : S35-43.

26. Van den Hout $H$, Reuser AJJ, Vulto AG, et al. Recombinant human alpha-glucosidase from rabbit milk in Pompe patients. Lancet $2000 ; 356$ : 397-8.

27. Klinge L, Straub U, Neudorf J, et al. Safety and efficacy of recombinant acid alpha-glucosidase (rhGAA) in patients with classical infantile Pompe disease : results of a phase II clinical trial. Neuromuscul Disord $2005 ; 15: 24-31$.

28. Van den Hout JM, Kamphoven JH, Winkel LP, et al. Long-term intravenous treatment of Pompe disease with recombinant human alpha-glucosidase from milk. Pediatrics 2004 ; 113 : e448-57.

29. Raben N, Fukuda T, Gilbert AL, et al. Replacing acid alpha-glucosidase in Pompe disease : recombinant and transgenic enzymes are equipotent, but neither completely clears glycogen from type II muscle fibers. Mol Ther $2005 ; 11: 48-56$.

30. Desnick RJ. Enzyme replacement and enhancement therapies for lysosomal diseases. J Inherit Metab Dis $2004 ; 27: 385-410$.
TIRÉS À PART

D.P. Germain 


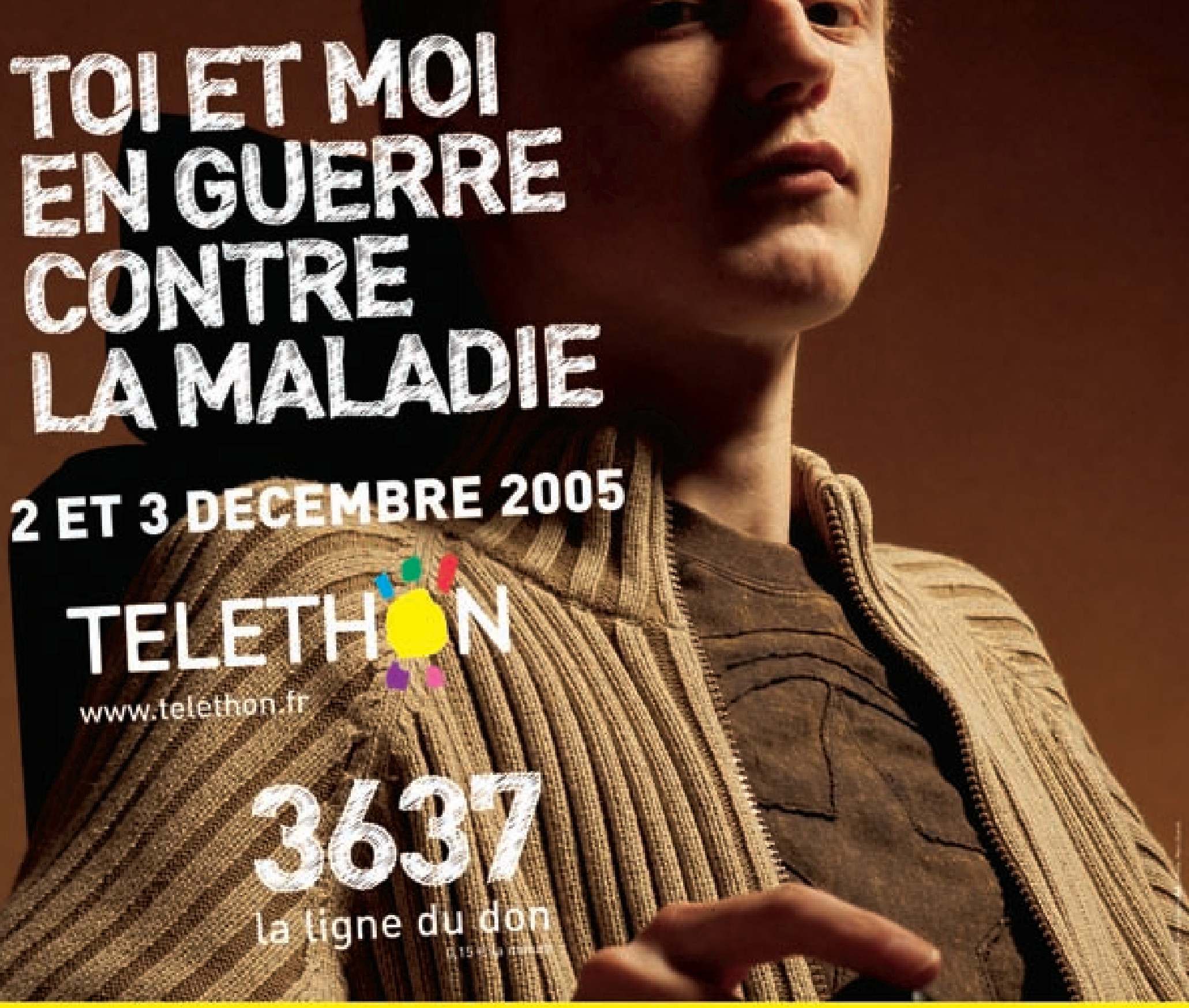

LA GUERRE CONTRE LA MALADIE, C'EST TOUS LES JOURS.

Les 2 et 3 décembre prochains sont deux jours de mobilisation qénérale. C'est le week-end du Téléthon, le week-end pour rejoindre le combat sur le front de la maladie. 